Preprints of the

Max Planck Institute for

Research on Collective Goods

Bonn 2006/16

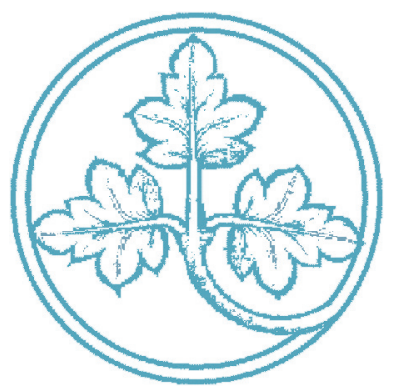

Mobile termination and collusion, revisited

Felix Höffler

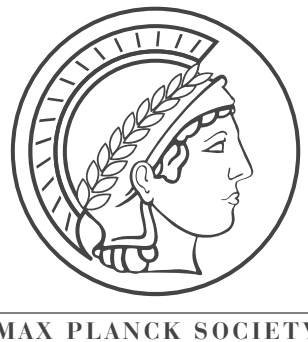




\section{Mobile termination and collusion, revisited}

Felix Höffler

June 2006

Max Planck Institute for Research on Collective Goods, Kurt-Schumacher-Str. 10, D-53113 Bonn http://www.coll.mpg.de 


\title{
Mobile termination and collusion, revisited
}

\author{
Felix Höffler*
}

June 26, 2006

\begin{abstract}
The standard model by Laffont, Rey and Tirole (1998) treats termination fees as an instrument to increase market power in a one-shot game of horizontal product differentiation. We offer an alternative view in an infinitely repeated Bertrand competition. We focus on symmetrical calling patterns and investigate simple two-part tariffs for two types, as well as general non-linear tariffs for two types and for a continuum of types. In this framework, termination fees make deviations from the collusive outcome less attractive. The optimum deviation strategy is usually to try to attract the high valuation customers since they exhibit the highest profits. Thus, a deviator will have a pool of high users which will have more outgoing than incoming calls, implying net termination payments. A cooperatively chosen termination rate can increase the deviator's cost and thereby always stabilizes collusion.

JEL-Classification:

Keywords: Two way access, mobile telecommunications, non-linear tariffs.
\end{abstract}

${ }^{*}$ Max Planck Institute for Research on Collective Goods, Kurt-Schumacher-Str. 10, 53113 Bonn, Germany. Phone: +49(0)228-9141646. hoeffler@coll.mpg.de. I would like to thank Stefan Behringer, Christoph Engel, Martin Hellwig, Hendrik Hakenes, Klaus Schmidt and Carl Christian von Weizsäcker for helpful discussions. Any remaining errors are mine. 


\section{Introduction}

There is a long discussion on excessive mobile termination rates, at least in Europe, under the calling-party-pays principle. This has led to numerous investigations of regulators (see e.g. Competition Commission (2002), Ofcom (2003)) and finally to European legislation, forcing each regulator explicitly to investigate and regulate the market for mobile termination (European Commission (2003)). That regulators need to control termination fees suggests that mobile operators have an interest in relatively high termination rates (see (IRG, 2002, 11) and (IRG, 2004, 33) for an assessment of the termination fees in Europe by the Independent Regulators Group).

This interest might be partially due to the fact that non-reciprocal termination fees between mobile operators on the one hand and fixed line operators on the other serve as a means for the mobile sector to exploit the fixed line sector. However, since, at least in Europe, all telecommunications incumbents are (or in case of British Telecom: were) integrated players it is unlikely that this is the only explanation (integrated players could oppose, via their mobile operations, coordination on termination fees that are only targeted at exploiting their own fixed line business). Thus, there is a strong suspicion that termination fees are used as an anti-competitive device in the mobile market.

This has given rise to a large, mainly theoretical, literature on twoway access. Seminal articles are by Armstrong (1998), Laffont, Rey, and Tirole (1998a) and Laffont, Rey, and Tirole (1998b) (A-LRT). The base model developed by A-LRT has been fruitfully used to investigate many additional aspects of the problem. Reviews of this literature can be found in Laffont and Tirole (2000), Armstrong (2002), and Vogelsang (2003). A short presentation focused on the problem of collusion, is found in Table 1 of Peitz, Valetti, and Wright (2004).

So - why another (theoretical) paper on this topic? The reason is that we depart from the A-LRT framework and focus on a different mechanism for collusion. In the A-LRT framework the question of collusion is posed in the following way: Do firms have an incentive to coordinate on termination fees above marginal cost, while competing in the retail market? We take the term collusion more literally, since, if firms cooperate, they might also collude in the retail market. For instance, in December 2005, the French competition authority found the three national mobile operators guilty of collusion and imposed a fine of $€ 534$ million, equivalent to about $3.5 \%$ of 
annual revenue of the companies involved. ${ }^{1}$ Thus, our question is: If firms want to collude in the retail market, can this be facilitated by excessive termination fees?

This alternative approach offers two additional contributions. First, we treat mobile voice communication as a homogenous good. Thus, we need not rely on "sufficient" horizontal product differentiation, which is vital for ensuring the existence of equilibria in the A-LRT model. We believe that - at least in the absence of the explicitly modeling of investments in branding - it is hard to imagine preferences for a particular operator. In our framework, equilibria in which termination fees support collusion always exist, even without horizontal product differentiation.

Second, we analyze non-linear tariffs for heterogeneous consumers and retain the result that termination facilitates anti-competitive behavior. Since it is obvious that, in the industry, firms do not charge linear tariffs, but rather offer complicated tariff schemes, there are many papers in the literature which account for this. However, in the A-LRT framework, and a couple of extensions, firms cannot gain from cooperatively chosen high termination fees if they charge non-linear tariffs in the retail market (noted already by Laffont, Rey, and Tirole (1998a), Proposition 7, and generalized for heterogeneous customers by Dessein (2004a) and Hahn (2004)).

These "neutrality results" are irritating, given the many complaints about and regulatory actions against overpriced termination fees. In contrast, in the approach of this paper, we find that with non-linear tariffs, termination fees above marginal cost (but not "too excessive"), will always facilitate collusion if the number of different consumer types is large. Our approach argues that as long as a monopolist makes higher profits on "high types" (which seems plausible and in fact occurs under standard assumptions of the single crossing property and the monotone hazard rate property), termination fees can always serve anticompetitive purposes by facilitating collusion.

The basic idea is that high termination fees make competitive undercutting strategies less profitable. This might best be illustrated by an example. Consider the situation of a small mobile operator who wants to increase his

\footnotetext{
${ }^{1}$ The allegation was, however, not directly on a price cartel but on a "Yalta on market shares" via exchange of strategically important information. However, it is difficult to imagine how to freeze market shares in this industry, where all firms serve all customer groups, whithout implicitely agreeing not to compete too fiercly on prices. The decision can be found at http://www.conseil-concurrence.fr/ pdf/ avis/ 05d65.pdf with an English summary http://www.conseil-concurrence.fr/ user/ standard.php?id_rub=160\&id article $=502$
} 
market share in order to better utilize his infrastructure. The operator could make calls cheaper in order to attract additional customers. However, with lower prices, his customers will make more calls and therefore also more outgoing calls than the customers of the other operators with higher prices. Thus, the operator with the low price will have to pay net termination fees to the competitors. This discourages price cuts and deviations from a collusive price level.

We capture this idea in a simple dynamic framework of collusion. $n$ firms play an infinite repetition of a Bertrand game in non-linear tariffs. Calls are produced at constant marginal cost. Firms have a capacity constraint such that they cannot, from one period to the next, increase capacities to serve all customers. No price discrimination between on- and off-net calls is possible. Consumers are heterogeneous in their marginal valuation for outgoing calls and receive no utility from incoming calls. They exhibit uniform calling patterns, i.e. everybody calls everybody else with the same probability. Collusion in this framework means that firms charge the non-linear tariff that maximizes industry profits as long as no one deviates. After deviation, they will punish (by playing a zero profit equilibrium of the stage game) forever.

Our main finding is that introducing termination fees above marginal cost (as long as they are no too large) will always facilitate collusion in this framework; i.e., collusion will be sustainable for lower values of the discount factor (see Ivaldi, Jullien, Rey, Seabright, and Tirole (2003) for a similar approach to collusion and the terminology of "facilitating collusion"). The reason is simple. A deviator must give customers at least the same utility as they are offered under the collusion tariff. Since the collusive offer already maximizes profits, the deviator can do no better than to copy this tariff. And since in the collusive tariff higher types provide higher profits, optimum deviation implies contracting with high types only. But high types make more than the average number of outgoing calls, implying net termination costs for the deviator when termination exceeds the marginal cost.

While this basic mechanism is intuitive, it is not trivial. In the example given above, one might propose that the deviator could, in non-linear tariffs, offer contracts with a high variable cost (which reduces calls and termination fees for the operator), which customers still accept due to low fixed fees or even subsidies. Even more, it can be shown that, as long as the deviator wants to serve the same types of consumers as the cartel, termination fees do actually increase deviation profits, which makes collusion more difficult to sustain. However, this can happen only if the number of different types is small. When the number of types becomes large, and can be approximated 
by a continuum of types, this effect disappears. The reason is that the deviator will always want to serve a different clientele than the cartel. Since he has a capacity constraint, he wants to serve only high types. But high types make more calls, implying net termination payments and a negative effect on the deviator's profits.

The remainder of the paper is organized as follows. Section two introduces the basic model. Section three analyzes a two-type model where firms are restricted to offering only a single, two-part tariff. Section four shows that the main insights easily carry over to the case where firms can make arbitrary non-linear offers. While in the two-type model, termination facilitates collusion only for some distributional assumption for the types, Section five, by analyzing a model with a continuum of types, shows that this restriction is an artefact of the two-point distribution. Section six concludes and discusses some additional aspects that are not modeled and how they might affect our argument.

\section{Model}

We investigate a market for a homogenous product (outgoing mobile voice calls), $q$. Consumers have quasilinear utility functions and gain utility only from making calls, not from receiving them. Let $t$ denote the total payment from the consumer to the firms, then the utility of a consumer of type $\theta$ is given by:

Assumption 1 Quasilinear utility

$$
\begin{aligned}
U_{\theta} & =u(q, \theta)-t, \\
u(0, \theta) & =0 \forall \theta, \\
u_{\theta}^{\prime} & \geq 0, u_{\theta}^{\prime \prime}<0, \quad \exists \bar{q}_{\theta} \forall \theta: u_{\theta}^{\prime}\left(\bar{q}_{\theta}\right)=0 .
\end{aligned}
$$

The reservation utility is independent of the type and normalized to zero. There is a satiation level $\bar{q}$ for all types, such that additional phone calls add no utility (but potentially disutility). Higher types value the good more. We assume the single crossing property for consumers' preferences.

Assumption 2 Single Crossing Property

$$
u_{\bar{\theta}}(q)>u_{\underline{\theta}}(q) \text { and } u_{\bar{\theta}}^{\prime}(q)>u_{\underline{\theta}}^{\prime}(q) \text { for } \bar{\theta}>\underline{\theta} \text {. }
$$

Furthermore, we assume uniform calling patterns. 
Assumption 3 Uniform calling pattern: A consumer making q outgoing calls will spread these calls evenly across all other consumers.

This implies that consumers that make more than the average number of outgoing calls will have more outgoing than incoming calls. ${ }^{2}$ It is also important to note that this implies that the "call balance" of any firm, i.e. the difference between outgoing and incoming calls, is always zero if one's own customers make the same number of outgoing calls as the average of the customers of all the other firms (independent of the market share of consumers). We assume that the number of consumers is large and without loss of generality normalize it to one.

There are $n>2$ symmetrical firms providing mobile services. All have installed a network, and network costs are sunk. The marginal cost of producing calls, in particular of terminating calls, is constant and normalized to zero.

Assumption 4 Constant marginal cost.

Firms face, however, a capacity constraint with respect to the number of customers they can serve.

Assumption 5 Capacity constraint: No firm can serve more than $\beta$ customers:

$$
\frac{1}{n-1}<\beta<1
$$

The assumption that $n-1$ firms can serve all consumers is crucial to support the competitive equilibrium in the Bertrand stage game, and might be violated in some mobile markets. While in our model all firms are symmetric, this is obviously not the case in reality. Thus, in some markets, dominant firms might have more than fifty percent of all subscribers. However, in western Europe, this is the case only in Belgium, Ireland and Portugal (EU, 10th Implementation Report, Annex 3, p. 52). This assumption also implies that in any (symmetric) equilibrium, firms will carry excess capacity. Although we do not model capacity decisions, a series of papers have shown that firms can have an incentive to carry excess capacity in order to be able to support collusion later on (Benoit and Krishna (1987), Davidson and Deneckere (1990)).

The second part of the assumption is also important but actually stronger than necessary for our results. We need to preclude that, from one period

\footnotetext{
${ }^{2}$ Dessein $(2004 b), 324$, reports from data supporting this assumption.
} 
to another, capacities can be expanded such that one player can serve the whole market. ${ }^{3}$

Firms compete in prices in a Bertrand fashion. In each period, all firms simultaneously announce (non-linear) prices. Afterwards each customer chooses the tariff offer that maximizes his or her utility. This interaction is infinitely repeated. Firms discount future profits at a discount factor $\delta$.

Termination fees $\tau, \tau \geq 0,{ }^{4}$ are reciprocal and constant over all periods. Termination payments are $\tau$ times the number of off-net calls, i.e. calls to customers of a different operator.

\section{Two types and two-part tariffs}

There are two types of customers. The share of high valuation types $\bar{\theta}$ equals $\alpha$; the share of low valuation types $\underline{\theta}$ equals $(1-\alpha)$.

Stage Game Firms are restricted to setting a single two-part tariff $(p, f)$ in each period, where $p$ denotes the variable per minute price and $f$ the fixed fee. In order to establish a Nash Equilibrium in the repeated game, we first derive the (worst) equilibrium of the stage game, which will be played after deviation.

Lemma 1 The unique symmetric equilibrium of the stage game is $p=0$ and $f=0$.

Proof. Given that all $n-1$ firms offer $p=0$ and $f=0$, a deviator cannot attract any customers without making losses, since $p=0$ maximizes the gains from trade which, under the claimed equilibrium, accrues wholly to the consumer. Thus, attracting consumers with $p$ or $f$ below zero inextricably leads to losses. Offering either $p$ or $f$ larger than zero would leave him with zero demand. There is no other equilibrium, since in any symmetric equilibrium there are idle capacities, which a firm could use by offering $\widetilde{p}-\varepsilon$ or $\widetilde{f}-\varepsilon$ and then supplying up to capacity, which is profitable for $\varepsilon$ small enough.

Collusion Consider a collusion equilibrium which takes this stage game as the punishment equilibrium once a firm has deviated from the collusive

\footnotetext{
${ }^{3}$ For the application to mobile markets, we think of transitory limitations with respect to sales personnel, customer care or procurement of mobile handsets.

${ }^{4}$ Restricting attention to termination fees above marginal cost does not qualitatively affect our results. We comment on this in the conclusions. In practice, regulators usually want $\tau$ to be "cost based", i.e. reflect some form of "long run incremental cost". Thus, marginal cost tend to be the lower bound for termination in practice.
} 
outcome. The collusive outcome maximizes industry profits and is shared evenly among all firms. If $(1-\alpha)$, the fraction of low types is sufficiently large, the maximum profits are the solution to the following maximization problem:

$$
\begin{array}{cl} 
& \max _{p, f} \bar{q}(p) p+f, \\
\text { s.t. }: & u_{\underline{\theta}}\left(q_{\underline{\theta}}(p)\right)-p q_{\underline{\theta}}(p)-f \geq 0,
\end{array}
$$

where $\bar{q}$ denotes the average consumption per customer:

$$
\bar{q}=\alpha q_{\bar{\theta}}(p)+(1-\alpha) q_{\underline{\theta}}(p) .
$$

The optimization problem reduces to:

$$
\max _{p} \bar{q}(p) p+u_{\underline{\theta}}\left(q_{\underline{\theta}}(p)\right)-p q_{\underline{\theta}}(p) .
$$

Call $\left(p^{*}, f^{*}\right)$ the solution to this problem and $\pi_{\text {all }}^{*}$ the average profit per customer resulting from $\left(p^{*}, f^{*}\right)$.

If there are few low types, i.e. $\alpha$ is larger than some cutoff value $\alpha^{*}$, then only the high types get served, and the optimum offer is $p=0$ and $f=u_{\bar{\theta}}\left(q_{\bar{\theta}}(p=0)\right)$, resulting in an average profit per customer of $\pi_{\bar{\theta}}$. The profits from collusion for a single firm therefore are:

$$
\Pi^{*}=\left\{\begin{array}{l}
\frac{1}{n} \frac{\pi_{a l l}^{*}}{1-\delta} \text { if } \alpha \leq \alpha^{*} \\
\frac{\alpha}{n} \frac{\pi \bar{\theta}}{1-\delta} \text { if } \alpha>\alpha^{*} .
\end{array}\right.
$$

For the profit maximizing collusive outcome, obviously, termination fees do not play a role. Due to our assumption of uniform calling patterns, each of the colluding firms that share the market evenly has the same number of customers and the same customer characteristics. Call $\widetilde{\Pi}$ the profit from an optimum deviation. It consists of the profit from the deviation period only, since, after the deviation period, profits will be zero forever. Hence, a collusion equilibrium exists only if:

$$
\frac{\Pi^{\text {Coll }}}{1-\delta} \geq \widetilde{\Pi} .
$$

Clearly, the standard result applies: if only firms are sufficiently patient, then collusion can always be supported. Our interest is whether termination fees can increase the range of values of $\delta$ in which collusion is an equilibrium. We therefore analyze the effect of termination fees on the deviation profits. 


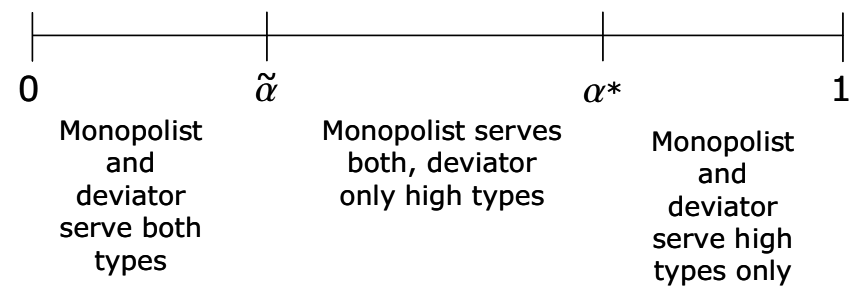

Figure 1: Different behavior of collusive firms and a deviating firm

Any reduction in $\widetilde{\Pi}$ will imply that (5) holds more easily, and therefore collusion can be supported even for lower values of $\delta$.

Deviation If a deviating firm were able to gain the whole market for some time before the competitors could react, then termination would not play a role. The deviator would have won all customers for this period, therefore all calls would be on-net. Since it is not realistic that a company will win all the customers before the competitors can react, we have introduced the assumption (3), stating that no firm can serve all customers. Thus, a deviator will also have off-net calls, and, as a result, termination fees might become relevant.

The impact of termination fees on the deviation profit depends crucially on whether the deviator serves the same type of customers or wants to serve a different customer pool. Given a capacity constraint, and in the absence of termination fees, there is always a parameter region of $\alpha$ such that, in the collusive solution $\left(p^{*}, f^{*}\right)$, both types of customers are served, while the deviator wants to serve only the high types. Figure 1 shows the different parameter constellations that are possible.

Lemma 2 If in the collusive outcome both types are served, and the capacity constraint (3) holds, there always exist values $\alpha \in\left(\widetilde{\alpha}, \alpha^{*}\right)$, such that optimal deviation implies that only high types $\bar{\theta}$ are served.

Proof. See Appendix.

Since the deviating firm has a capacity constraint, it does not want to "waste" scarce capacity on low return customers. Thus, it will abstain from serving low types even for a lower value of $\alpha$. This is most obvious in the case where $\beta \leq \alpha$. A deviator cannot serve more than the high types. Thus, he will never waste capacity on low profit $\underline{\theta}$ types. We will later show that an interval in which the deviator prefers to serve only high types, while 
the cartel serves both, also exists with termination fees and that under termination fees this interval is even larger.

Let $\bar{q}(p)$ denote the average consumption per customer having accepted an offer $(p, f)$. A deviator offering a tariff $(\widetilde{p}, \tilde{f})$ and serving a fraction $\widetilde{\beta} \leq \beta$ of all customers will - in the deviation period - make a profit of:

$$
\widetilde{\pi}=\widetilde{\beta}\left[\bar{q}(\widetilde{p})(\widetilde{p}-\tau)+\tau\left((1-\widetilde{\beta}) \bar{q}\left(p^{*}\right)+\widetilde{\beta} \bar{q}(\widetilde{p})\right)+\widetilde{f}\right] .
$$

The term in the square brackets equals the profit per customer. The first product $\bar{q}(\widetilde{p})(\widetilde{p}-\tau)$ reflects the call revenues, net of the termination fees $\tau$. Note that this includes termination fees of "on-net calls", i.e. payments made from the deviator to himself. The second term reflects the termination revenues. Given the assumption of uniform calling patterns and a large population, these are (almost) identical for each customer, independent of his own calling pattern. ${ }^{5}$ Again, these include the revenues stemming from termination payments the deviator makes to himself (the term $\widetilde{\beta} \bar{q}(\widetilde{p})$ ). Finally, the deviator receives the fixed payment $\widetilde{f}$ per customer who signs the contract.

It is straightforward that whenever the deviator has the same customer base as the cartel, he will never be worse off by introducing termination fees. He can always mimic the collusion contract and, by so doing, avoid any net payments of termination fees. This is due to our symmetric calling pattern assumption. The deviator can, however, do better, since the termination fees introduce a new source of revenues. By increasing the variable price $p$, he gets net termination payments since his customers will make less outgoing calls than the customer who stayed with the cartel.

\footnotetext{
${ }^{5}$ Note that this is approximately true only if the population is sufficiently large. Call $m$ the number of customers. Taking into account that one does not call oneself, the exact formulation would be as follows: an $h$-type receives

$$
(\alpha m-1) \frac{q_{h}}{m-1}+(1-\alpha) m \frac{q_{l}}{m-1}
$$

incoming calls, and an $l$-type receives

$$
\alpha m \frac{q_{h}}{m-1}+((1-\alpha) m-1) \frac{q_{l}}{m-1}
$$

incoming calls. Both expressions converge to the to $\bar{q}=\alpha q_{h}+(1-\alpha) q_{l}$ for $m \rightarrow \infty$, as used in (6). Note that for $m$ small, this approximation is bad. For example, consider $m=2$, where the first consumer makes 2 calls and the second 0 calls. The total number of calls equals two, but the first consumer has zero incoming calls, the second two incoming calls.
} 
Proposition 1 The introduction of a termination fee $\tau>0$ (i) does not facilitate collusion for $\alpha \in(0, \widetilde{\alpha}]$ and (ii) makes collusion more difficult to sustain for $\alpha \in\left[\alpha^{*}, 1\right)$.

Proof. See Appendix.

Although the tariff offered by the deviator reduces the total surplus per customer served by the deviator, the deviator can exploit the other firms. Termination therefore makes deviation more profitable if the customer pool of the deviator and of the collusive outcome have the same characteristics. This effect is similar to what is often discussed in the context of fixed versus mobile termination. It has been frequently argued that the mobile operators exploit the fixed line companies by setting unilaterally high termination fees (see Armstrong (2002), 337-341, for a theoretical treatment).

Now consider the intermediate case $\alpha \in\left(\widetilde{\alpha}, \alpha^{*}\right)$, where the cartel serves both types while a deviator finds it optimal to serve high types only. In the absence of termination fees, the deviator would set $p=0$ and extract all rent, except for the high types' information rent under the collusive outcome. This would, however, imply a non-equalized call balance. The deviator's customers would have more outgoing than incoming calls. Thus, when introducing termination fees, the deviator ends up with net payments for termination fees (an "access deficit" in A-LRT terminology). Therefore, introducing termination fees - as long as they are not too large - will always reduce the deviator's profits and make collusion easier to support.

Proposition 2 If $\alpha \in\left(\widetilde{\alpha}, \alpha^{*}\right)$, there exists a cutoff value $\widehat{\tau}$ such that the introduction of a termination fee $\tau \leq \widehat{\tau}$ facilitates collusion in the sense that collusion is not sustainable for values of $\delta$ for $\tau=0$, while it is sustainable for $0<\tau \leq \widehat{\tau}$. Deviation profits are minimized at $\tau=\widehat{\tau}$

Proof. See Appendix.

Figure 2 illustrates the effect of termination fees on the revenues from customers. Here, $q$ denotes the quantity consumed by a certain customer. Instead of supplying the maximum quantity to the high type (at the high type's utility level attainable from the collusion offer), the deviator chooses a positive price to reduce the high type's demand in order to save on termination fees (the slope of the offer is positive). The deviator's profits are here lower than they would be in the absence of termination fees.

This highlights the general trade-off for the deviator. He can generate income from customers or (via termination revenues) from competitors. If he sets a low usage fee, he will be able (via the fixed fee) to get a high 


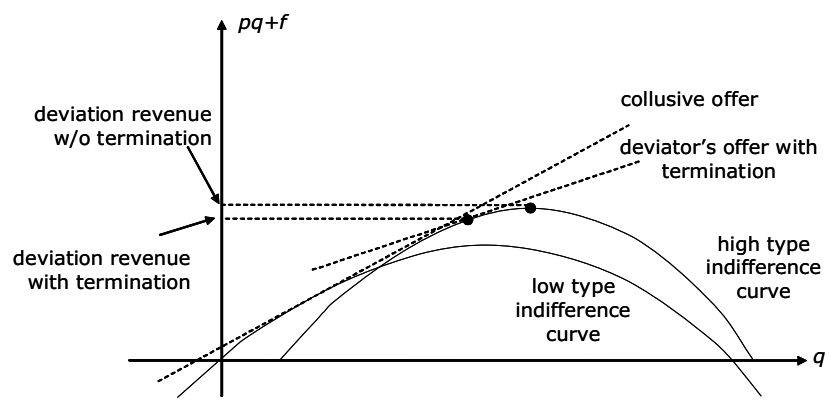

Figure 2: Termination with two-part tariff and two types

total payment from the customers, since he increases the attainable surplus by setting the price equal to the marginal cost. With termination fees, this strategy leads to net termination payments to competitors. Setting a higher variable price reduces revenues from customers, but also payments to competitors. If the termination fee becomes very large, it will be optimal for the deviator to set a very high variable price (and possibly serve only low types) and to make his profit on the termination payments he will receive from the competitors. Therefore, only termination fees "moderately" above marginal cost can facilitate collusion.

Provided with Proposition 2, we can now validate that an intermediate interval $\left(\widetilde{\alpha}, \alpha^{*}\right)$ of values of $\alpha$ always exists in which a deviator would like to serve only high types, while the cartel serves both types. Lemma 2 showed this for the case of $\tau=0$, and we need now to assure that the same holds for $\tau>0$. Start by assuming that $\tau=0$. Then we know from Lemma 2 that $\widetilde{\alpha}<\alpha^{*}$. At $\widetilde{\alpha}$ the deviator is indifferent between serving both or only high types. Now introduce termination $\tau>0$. From Proposition 2 we know that at $\widetilde{\alpha}$, where the deviator serves only high types, but the monopolist serves both, termination reduces profits. At $\widetilde{\alpha}$ it will now be strictly better to serve only high types; thus the cutoff is even below $\widetilde{\alpha}$.

If, however, for some other reason not covered in this section's model (e.g. to exploit the fixed line sector), $\tau$ is set relatively high, the collusion outcome might be affected by $\tau$ in an unexpected manner. High termination fees can be a motivation for the cartel to set the collusion price even above the monopoly price $p^{*}$ that maximizes industry profits.

Proposition 3 If both types get served in the collusion outcome and only the high types get served in the deviation outcome, there can be values of 
$\tau \in(\underline{\tau}, \bar{\tau})$ such that collusion will be easier to sustain if the collusion offer is $(\widehat{p}, \widehat{f}), \widehat{p}>p^{*}, \widehat{f}<f^{*}$. Sufficient conditions for this are (i) that the demand functions of the types are close, $\bar{q}(p)=\underline{q}(p)+\varepsilon$, and (ii) that they are sufficiently convex, $q(p)^{\prime \prime}$ sufficiently large.

Proof. See Appendix.

Consider a case where $\delta$ is so low that the cartel cannot sustain collusion when realizing the monopoly solution. To make collusion possible, it has to drive a wedge between the collusive payoff and the deviation payoff. We have shown before that it can do so by increasing $\tau$ above zero. Now imagine that it cannot influence $\tau$ (or $\tau$ might already be set at $\tau^{*}$ and collusion is not sustainable). It can never increase the collusion payoff any further, and we have already assumed maximum punishment after deviation. The only alternative left is to alter the collusion tariff such that the deviation profit decreases. Increasing $p$ above $p^{*}$ can serve this purpose. On the one hand, this clearly reduces the collusive payoff, which is bad for sustaining collusion. On the other hand, raising $p$ lowers the number of outgoing calls of the customers of the cartel. Thus, if a deviator has a customer basis which makes more calls than the customer basis of the cartel, then this will increase the imbalance of outgoing and incoming calls. And, if $\tau$ is large, this hurts the deviator so much that it overcompensates for the negative effect on the collusive payoff; consequently, the difference between the collusion payoff and the deviation payoff increase.

However, if $\tau$ is too large, the optimum deviation will no longer be to serve only high types. For very high $\tau$ it will be optimal to deviate to serving only low types and to make profits mainly on the termination fees. Thus, it is only for intermediate values of $\tau$ that the effect of Proposition 3 can occur. The interval $(\underline{\tau}, \bar{\tau})$ is non-empty if switching to serve only low types is very unattractive because this will sharply decrease payments from customers, while, at the same time, both types make approximately the same number of calls at the relevant price levels. This happens if both demand functions are close and very convex.

If the effect of Proposition 3 occurs, the termination fees introduce an additional welfare problem since the collusion outcome does worse than the monopoly outcome. The cartel increases price above the monopoly level just in order to prevent profitable collusion. 


\section{Two types and general non-linear tariffs}

We just want to discuss briefly what happens if firms are not restricted to offering a single two-part tariff but can offer any number of two-part tariffs or even general non-linear tariffs. Termination fees can still reduce the deviation profit, and they therefore support collusion in this context.

First note that offering $p=0, f=0$ is again an equilibrium in the stage game. Gaining positive payments from customers would always make them worse off since they already achieve the maximum utility from consuming the good at $p=0$ and $f=0$. And any firm would loose all customers when demanding positive payments since $n-1$ firms can serve all customers.

Second, there will be values of $\alpha$ and $\beta$, such that in the collusive outcome, all types get served while a deviator serves only high types. At least this will be the case for $\beta \leq \alpha$. Again, the reason is that profits are higher on higher types and therefore the deviator does not want to "waste" his scarce capacity on serving the low valuation customers. That profits are higher on customers who exhibit a higher willingness to pay is not only intuitive, it is also a general property of "well behaved" adverse selection problems. Therefore, and for further use in the next section, we state this more formally in the following Lemma. Note that the results hold for any number of types as well as for a continuum of types.

Lemma 3 Call $\left(t^{*}(\theta), q^{*}(\theta)\right)$ the non-linear contract that maximizes industry profits under the assumptions 1 (concave, quasilinear utility and type independent reservation utility) and 2 (single crossing property). Then profits per customer are non-decreasing in types and strictly increasing in types if $q^{*}(\theta)$ strictly increases in $\theta$.

Proof. See Appendix.

The intuition for this result becomes clear from analyzing a discrete number of types. The proof is by contradiction. Were the profit on a type $\bar{\theta}$ lower than on the next lower type $\underline{\theta}<\bar{\theta}$, then a monopolist offering $\left(t^{*}(\theta), q^{*}(\theta)\right)$ could just cancel the offer $\left(t^{*}(\bar{\theta}), q^{*}(\bar{\theta})\right)$. Due to the single crossing property and its implication that, in the optimum, the incentive compatibility constraints are downward binding, the high type would choose the low type's offer. By doing so, the monopolist could increase his profit, a contradiction to the claim that $\left(t^{*}(\theta), q^{*}(\theta)\right)$ is profit maximizing.

Coming back to the case of two types, we note that, if the collusive outcome serves both types, the solution will have the standard properties: the high type will consume the optimum quantity (no distortion at the 


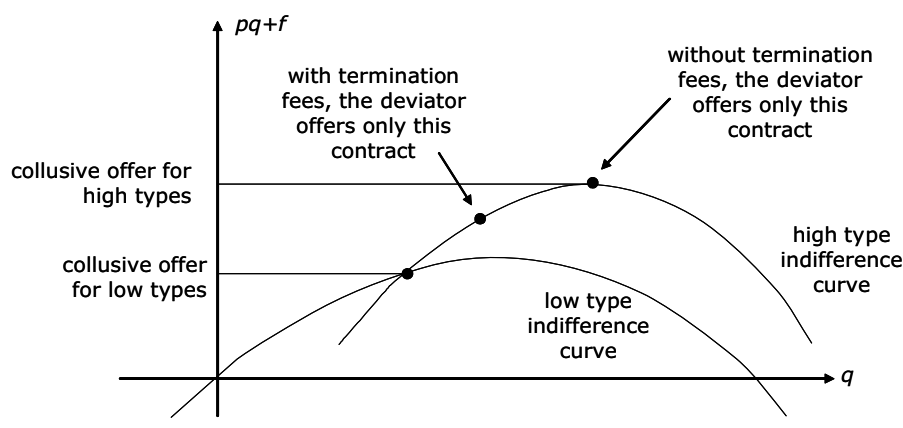

Figure 3: Termination with general non-linear tariffs and two types

top), while the low type will have inefficiently low consumption and will receive only his reservation utility. A deviator who serves only the high types will mimic the collusive offer for the high types (minus perhaps some $\varepsilon)$ and, therefore, end up with a customer pool having more outgoing than incoming calls. The same logic applies as before: introducing a not-too-large termination fee will reduce the deviation profit by increasing the "access deficit". Figure 3 illustrates this effect.

\section{A continuum of types and general non-linear tariffs}

Our discussion so far has shown that, under certain assumptions on the type distribution (i.e. on the parameter $\alpha$ ), termination fees can facilitate collusion. For other parameter values of $\alpha$, termination hinders collusion. In this section we want to show that this ambiguity is an artefact of the two-type case and vanishes once the number of types becomes large.

In the two-type case, termination hindered collusion if the same customer base is served in the collusion outcome and the optimum deviation. Now consider that the number of types is $K$. The collusion outcome serves the $k$ highest types. When will the deviator find it optimal also to serve exactly the same $k$ highest types? Only for very specific combinations of the distributional assumption and the capacity constraint $\beta$ this will occur (types lower than $k$ must be rare or their valuation of the service must be very low, and $\beta$ must be relatively large, such that the deviator can indeed serve the $k$ highest types). This parameter constellation will become more 
and more special once the number of types grows (except for the case where there are about $\beta$ customers with very high valuations and the rest has very low valuations - which is again a sort of a two-type distribution). Thus, if the number of types grows, deviation will typically imply that a different customer pool is served by the deviator.

As a limit argument, we consider the case of a continuum of types, $\theta \in(0,1)$. The distribution function of agents is denoted by $P(\theta)$, with density $p(\theta)$. To facilitate the analysis, we want to focus on the case where the collusive outcome serves all customers. ${ }^{6}$ In order to rely on standard solutions for the resulting adverse selection problem (see e.g. Fudenberg and Tirole (1991), Chapter 7.3), we need some further assumptions:

Assumption 6 Monotone hazard rate property:

$$
\frac{\partial}{\partial \theta}\left(\frac{p(\theta)}{1-P(\theta)}\right) \geq 0
$$

Assumption 7 Utility function: The single crossing property holds, $\frac{\partial^{2} u(q, \theta)}{\partial q \partial \theta}>$ 0 and $\frac{\partial^{3} u(q, \theta)}{\partial q \partial \theta^{2}} \leq 0$ and $\frac{\partial^{3} u(q, \theta)}{\partial q^{2} \partial \theta} \geq 0$.

Assumptions 1, 3, 4, and 5 still hold. Firms compete in a Bertrand fashion by simultaneously announcing a non-linear tariff, specifying a transfer payment $t$ for each quantity $q$. The game is infinitely repeated.

If firms collude, they realize the maximum profit $\Pi^{\text {Coll }}$ and share this evenly as long as no firm deviates. If someone deviates, they will play a punishment equilibrium, yielding $\Pi^{\text {pun }}<\Pi^{\text {Coll }}$. Given our assumptions, the characterization of the profit maximizing (monopolistic) solution is well known. The monopolist specifies payments and quantities such that the quantities $q^{*}(\theta)$ and payments $t^{*}(\theta)$ are strictly increasing in the types $\theta$. By Lemma 3 , this also implies that the profit per type is increasing in $\theta$.

First note that an equilibrium in the stage game is that each firm announces a linear tariff with a price equal to marginal cost. This maximizes the gains from trade for each type and implies zero profits for the firms. Firms cannot profitably deviate: attracting customers from other firms would require giving them more than the maximum surplus - which implies losses for the firm. Providing the customers with less than the maximum

\footnotetext{
${ }^{6}$ It is easy to include the case where there is a type $\underline{\theta}$ such that the cartel also serves only types $\theta>\underline{\theta}$. In this case we need to make the assumption that no single firm can serve all these customers, i.e. $\beta<1-P(\underline{\theta})$.
} 
surplus would leave the deviating firm with no customers, since $n-1$ firms can serve the overall market. ${ }^{7}$

Thus, collusion can only be supported if the payoff from colluding forever exceeds the payoff from deviating in one period and earning zero forever:

$$
\frac{\Pi^{\text {coll }}}{1-\delta} \geq \Pi^{d e v}
$$

Due to our assumption on the capacity constraint, no deviator can serve the whole market, but only a fraction $\beta<1$. Again, what we want to preclude is that a firm can win the whole market from one period to the next. Therefore, if a firm deviates from the collusive outcome, it will be able to serve only a fraction of the total market. With no termination fees, $\tau=0$, optimum deviation requires that the deviator offers a "truncated contract": he mimics the contract of the collusion outcome (possibly plus some $\varepsilon$ benefit for the customers), but only for the highest types. The reason is again that high types generate more profit, and consequently the deviator does not want to waste his scarce capacity on low profit types.

Lemma 4 For $\tau=0$, a deviator offers a truncated contract with $q^{*}(\theta)$ and $t^{*}(\theta)$ for $\theta \geq \widetilde{\theta}$, where $\widetilde{\theta}$ is defined by $1-P(\widetilde{\theta})=\beta$.

Proof. See Appendix.

Figure 4 illustrates this Lemma. The function $q^{*}(\theta)$ is the solution to the monopolist's problem, i.e. the quantities for each type offered by the colluding firms. The deviator takes the quantity of the marginal customers $q(\widetilde{\theta})$ as the minimum quantity he is willing to supply and offers the same as the collusive offer for all other types (i.e. the same price-quantity combinations $(t, q)$ for $q \geq q(\widetilde{\theta}))$.

In the deviator's problem, the incentive compatibility constraint of $\tilde{\theta}$ of the monopolist's problem is just replaced by $\widetilde{\theta}$ 's participation constraint, i.e., $\widetilde{\theta}$ must receive at least as much utility from the deviator's offer as from the collusive outcome. Therefore, for all $\theta \geq \widetilde{\theta}$, the solutions are identical.

\footnotetext{
${ }^{7}$ Mandy (1992) shows that there may also exist equilibria with positive profits (since our model is a model without free entry). However, if we slightly alter the assumption on the capacity constraint (3) into $1 /(n-2)<\beta<1$, i.e. $n-2$ firms can serve the whole market, then we can always construct a punishment equilibrium with zero profits as the unique outcome. Among the non-deviating firms, one (randomly selected) firm will take the role of an entrant: it will be inactive as long as there is no profitable offer possible. Then Mandy's Properties FE1-FE5 apply and a uniform price equal to the minimum of average cost (which is constant in our case) is the unique Bertrand equilibrium.
} 


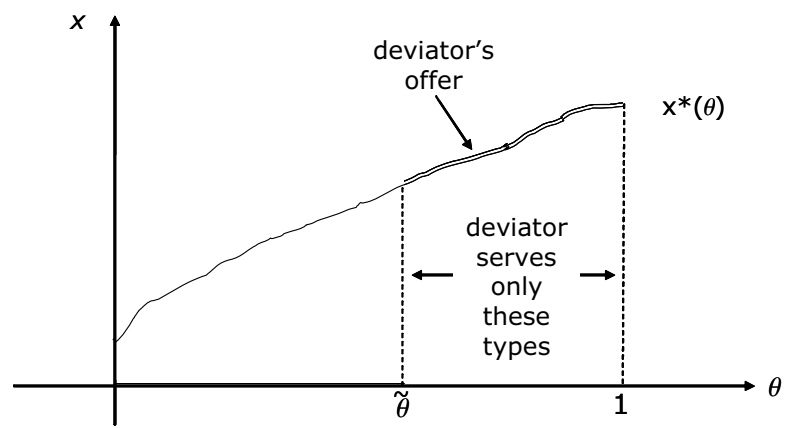

Figure 4: Optimum non-linear deviation without termination fees

The result of such a strategy is that the deviator will have a customer pool that makes more outgoing calls than incoming calls (which is just a consequence of $q^{*}(\theta)$ being increasing). Therefore, the introduction of a not-too-large termination fee $\tau>0$ will cause an "access deficit", i.e. net termination payments of the deviator to the other firms, and it will therefore reduce the deviation profits. This reduction in deviation profits makes collusion easier to support.

Proposition 4 With a continuum of types and non-linear tariffs, termination fees will always facilitate collusion, as long as they are not too large; i.e. there will be levels of the discount factor $\delta$ such that, without termination, collusion cannot be supported, while with termination it can be supported.

Proof. See Appendix.

The deviator faces a trade-off. When serving high types, he will receive high payments from the customers, but he will also have to make high termination payments to the other firms due to the imbalanced calling pattern (more outgoing than incoming calls). In order to receive at least as much profits as in the absence of termination fees, the deviator therefore would need to gain net termination income. He can do so by serving low types instead of high types, or offering contracts to high types under which they make fewer calls. Since $q^{*}(\theta)$ and $t^{*}(\theta)$ have been constructed such that they maximize the profits from any capacity $\beta, 0<\beta<1$, this would imply a discrete loss in payments from customers, which cannot be compensated if the termination fee is not very large. Therefore, the deviation profit will decrease when introducing a - not-too-large - termination fee. 
Once the termination fee becomes too high, the deviation profit might be increasing in $\tau$; it might even be higher than the deviation profit in the absence of termination (and thereby hinder collusion). If $\tau \rightarrow \infty$, it will obviously be optimal to serve only the lowest types and pay them not to make calls at all.

However, if firms can cooperatively set a common termination fee, they will probably avoid this. Our results rather show that they will always have an interest in setting a termination fee "moderately" in excess of marginal cost if they want to collude since this reduces the payoffs of a firm deviating from the collusive outcome.

\section{Conclusion}

This paper offers an explanation for why termination fees can support collusion in the retail market when mobile operators can cooperatively influence them. The key effect is that termination fees above marginal cost reduce the deviation profits. The optimum deviation strategy is usually to try to attract the high valuation customers since they are the ones with the highest profits. This strategy is made less attractive by setting termination fees above cost, since a deviator with a pool of high users will have more outgoing than incoming calls. The reduction in deviation profits stabilizes the collusion.

This is a complementary explantation to the one given by A-LRT, where termination fees can be used to "raise rivals' costs". Our approach, however, circumvents some of the problems of existence of equilibria in A-LRT and can easily show collusive effects when allowing for non-linear tariffs, which is difficult in A-LRT.

Our analysis has abstracted from many other aspects which are highly relevant and which result in highly complex mobile tariffs. Many mobile operators charge different prices for on- and off-net calls. In the A-LRT framework, Gans and King (2001) find that cooperatively choosing termination fees might serve anticompetitive purposes, but will be set below marginal cost. Allowing for such price discimination in our framework would make deviation easier under termination fees. The deviator could attract the high types by offering low priced on-net calls while off-net calls will be expensive. This reduces the negative effect of termination fees on the deviation profits. It does, however, not erase the negative effect of termination on collusion payoffs, since termination still adds an additional aspect the deviator has to accout for and which does not allow him to realize the maximum deviation 
profit possible in the absence of termination fees. ${ }^{8}$

Under non-uniform calling patterns, where some customers are more likely to be called than others, the basic effect of our model should still occur, as long as the calling-party-pays principle applies. In the collusive outcome, asymmetric calling patterns do not play a role because profits are shared evenly among all firms (all firms are symmetric and serve on average the same customer base). If the termination fee is not too large, a deviator would still try to focus on those customers that make many outgoing calls, since this is the primary source of revenue. Only with high termination fees, it might become interesting to attract only customers with far more incoming than outgoing calls, like call centers.

For expositional convenience we restricted attention to termination fees above marginal cost, i.e. $\tau>0$. Termination fees below marginal cost are equivalent to subsidizing firms with more outgoing than incoming calls. This would make deviation from the collusive outcome more attractive. Firms that want to use termination fees to support collusion would therefore never choose termination fees below marginal cost.

The key issue in our model is that, in the supergame, the deviator need not just mimic the collusive behavior minus epsilon. With differentiated consumers, he can make higher profits per customer or per unit sold compared to the collusive outcome. While the cartel will serve "many" customers, a deviator will typically only be able to serve fewer customers and therefore will concentrate on the most attractive ones; i.e., he can engage in "cherry picking". This might be of interest beyond its application to mobile termination, where the termination fee serves as a device to make cherry picking harder. Proposition 3 of our paper already points in an additional direction: the collusive outcome might not maximize industry profits. Rather, the cartel might need to forego profits in order to hamper cherry picking by deviators. It might try to lock in the most valuable customers (e.g. via air miles bonus programs), for cartel members have to invest something (lounges, free flights). But it ties the attractive customers to the cartel for a sufficiently long period such that a deviator will not make profits before the cartel can react and punish the deviation. This type of interaction between optimum deviation and optimum collusive behavior might be of interest for future research.

\footnotetext{
${ }^{8}$ One could even speculate whether the cartel might not also introduce price discrimination between on- and off-net calls. Then, a deviator would find it harder to attract customers just by making on-net calls cheaper.
} 


\section{Appendix}

\subsection{Proof of Lemma 2}

(i) Profits on high types are higher than on low types: both pay the same fixed fee $f$, and given the uniform price and constant marginal cost, per unit profits are the same, but high types buy more units, due to the single crossing property.

(ii) Therefore, the deviator's profit is non-decreasing in $\alpha$ (it strictly increases if the deviator serves both types, and remains constant if the deviator serves only high types).

(iii) If $\beta \leq \alpha$, the deviator will never serve low types: two-part tariffs with $f>f^{*}$ and $p<p^{*}$, which provide the same utility for the high type, will attract only high types, and profits per customer will be at least as high as under $\left(f^{*}, p^{*}\right)$, which, due to (i), increases profits (profits per customer are actually higher since the deviator can neglect the low type's participation constraint). Thus, at $\alpha^{*}$ the deviator is not indifferent but strictly prefers serving high types only.

(iv) If $\beta>\alpha$, the deviator might decide to serve only high types and leave some capacity idle. At $\alpha^{*}$ the monopolist is just indifferent between serving both types and serving only high types:

$$
\alpha^{*} \pi_{\bar{\theta}}=\alpha^{*} \pi_{\text {all }}^{\bar{\theta}}+\left(1-\alpha^{*}\right) \pi_{\text {all }}^{\theta} .
$$

At $\alpha^{*}$, the deviator can earn the same from serving only high types (namely, $\alpha^{*} \pi_{\bar{\theta}}$ ), but strictly less when serving all types, due to the capacity constraint (namely, $\beta\left[\alpha^{*} \pi_{\text {all }}^{\bar{\theta}}+\left(1-\alpha^{*}\right) \pi_{\text {all }}^{\underline{\theta}}\right]$ - neither can the deviator do better by altering $f$ or $p$, since $f^{*}$ and $p^{*}$ already maximize the profit when serving both types). Then, at $\alpha^{*}$, the deviator again strictly prefers to serve high types only. Due to (ii), $\widetilde{\alpha}$, the point of the deviator's indifference between serving both types or only high types, must be smaller than $\alpha^{*}$.

\subsection{Proof of Proposition 1}

The first part is obvious, since the deviator can always offer the same tariff as the cartel and thereby avoid any net termination payments. We need to show that the deviator is indeed strictly better off by introducing termination fees for $\alpha \in\left[\alpha^{*}, 1\right)$, i.e. the deviator, as well as the cartel, serves high types only. If the deviator wants to serve only the high types, he will maximize (6) subject only to the high type's participation constraint:

$$
\tilde{f} \leq u_{\bar{\theta}}\left(q_{\bar{\theta}}(\widetilde{p})\right)-q_{\bar{\theta}}(\widetilde{p}) \widetilde{p}-U_{\bar{\theta}}\left(p^{*}, f^{*}\right),
$$


where $U_{\bar{\theta}}\left(p^{*}, f^{*}\right) \geq 0$ denotes the information rent the high type receives in the collusion outcome. The deviator might use all of his capacity $\beta$ or only a part of it, $\widetilde{\beta} \leq \beta$. Using (8) the deviator maximizes:

$$
\begin{aligned}
& \max _{\widetilde{p}} \widetilde{\beta}\left\{\bar{q}(\widetilde{p})(\widetilde{p}-\tau)+\tau\left[(1-\widetilde{\beta}) \bar{q}\left(p^{*}\right)+\widetilde{\beta} \bar{q}(\widetilde{p})\right]\right. \\
& \left.+u_{\bar{\theta}}\left(q_{\bar{\theta}}(\widetilde{p})\right)-q_{\bar{\theta}}(\widetilde{p}) \widetilde{p}-U_{\bar{\theta}}\left(p^{*}, f^{*}\right)\right\} .
\end{aligned}
$$

Using from the customers optimization that $\frac{\partial u}{\partial q}=p$, the first order conditions imply:

$$
\widetilde{p}=(1-\widetilde{\beta}) \tau,
$$

i.e. the price equals the perceived marginal cost of the deviating firm. And - after some rearrangements - profits are given by:

$$
\widetilde{\pi}=\widetilde{\beta}[\underbrace{\tau(1-\widetilde{\beta})\left[\bar{q}\left(p^{*}\right)-q_{\bar{\theta}}(\widetilde{p})\right]}_{\text {deviator's termination revenue }}+\underbrace{u_{\bar{\theta}}\left(q_{\bar{\theta}}(\widetilde{p})\right)-\underbrace{U_{\bar{\theta}}\left(p^{*}, f^{*}\right)}_{\text {high type's information rent }}]}_{\text {payment from customer to deviator }} .
$$

Thus:

$$
\begin{aligned}
\frac{\partial \widetilde{\pi}}{\partial \tau} & =\widetilde{\beta}\left\{(1-\widetilde{\beta})\left[\left[\bar{q}\left(p^{*}\right)-q_{\bar{\theta}}(\widetilde{p})\right]-\tau(1-\widetilde{\beta}) q_{\bar{\theta}}^{\prime}\right]+\widetilde{p} q_{\bar{\theta}}^{\prime}(1-\widetilde{\beta})\right\} \\
& =\widetilde{\beta}(1-\widetilde{\beta})\left[\bar{q}\left(p^{*}\right)-q_{\bar{\theta}}(\widetilde{p})\right],
\end{aligned}
$$

which is positive, since $p^{*}=0$ (from the problem of maximizing cartel profits, note that also the cartel serves only high types here) and $\widetilde{p}=(1-\widetilde{\beta}) \tau>$ 0 for $\tau>0$.

\subsection{Proof of Proposition 2}

If both types get served in the collusive outcome, $p^{*}>0$, determined by

$$
\begin{aligned}
p^{*} & =\frac{1}{-\bar{q}^{\prime}}\left(\bar{q}\left(p^{*}\right)-q_{\underline{\theta}}\left(p^{*}\right)\right) \\
& =\frac{\alpha}{-\bar{q}^{\prime}}\left(q_{\bar{\theta}}\left(p^{*}\right)-q_{\underline{\theta}}\left(p^{*}\right)\right)>0,
\end{aligned}
$$

where $\bar{q}=\alpha q_{\bar{\theta}}(p)+(1-\alpha) q_{\underline{\theta}}(p)$. From (9) we know that, for $\tau$ sufficiently 


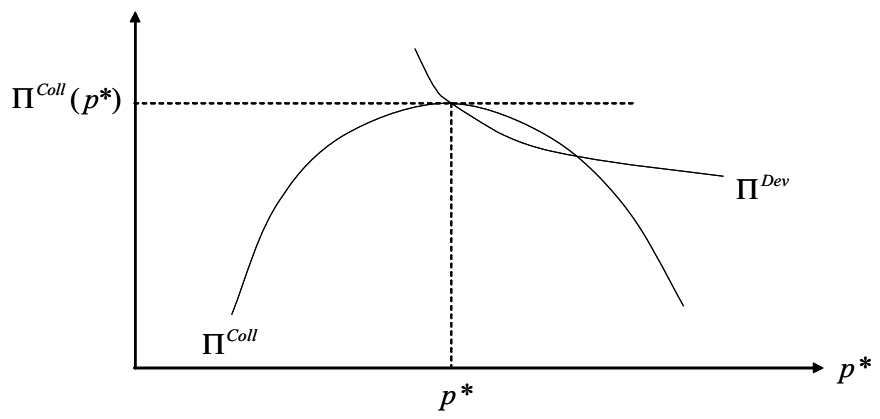

Figure 5: Wedge between deviation profit and collusion profit

small, $\widetilde{p}<p^{*}$. Since $q_{\bar{\theta}}(p)>q_{\underline{\theta}}(p)$, we also have $q_{\bar{\theta}}(p)>\bar{q}(p)$ for $\alpha<1$. Thus, for $\tau$ sufficiently small, $\tau \leq \widehat{\tau}, \widehat{\tau}>0, \bar{q}\left(p^{*}\right)<q_{\bar{\theta}}(\widetilde{p})$, which, by (11), implies that the deviation profit decreases in $\tau$. Therefore, deviation profits are minimized at $\widehat{\tau}$.

\subsection{Proof of Proposition 3}

Assume that maximizing industry profits requires serving both types while optimum deviation requires serving only high types. Consider the knife-edge case, where for given $\tau$ even with the profit maximizing contract $\left(p^{*}, f^{*}\right)$, a deviator earns just as much from deviating as from not deviating:

$$
\begin{aligned}
\Pi^{\text {Coll }}(\delta)= & \Pi^{\text {Dev }}(\tau) \\
\frac{1}{1-\delta} \frac{\widetilde{\beta}}{n} \pi\left(p^{*}\right)= & \widetilde{\beta}\left[(1-\beta) \tau\left[\bar{q}\left(p^{*}\right)-q_{\bar{\theta}}(\widetilde{p})\right]+u_{\bar{\theta}}\left(q_{\bar{\theta}}(\widetilde{p})\right)\right. \\
& \left.-U_{\bar{\theta}}\left(p^{*}, f^{*}\right)\right] .
\end{aligned}
$$

Now consider a marginal increase in $p^{*}$. We want to show that constellations exists such that a situation as in Figure 5 can arise: An increase of $p$ beyond the profit maximizing level $p^{*}$ can introduce a difference between the collusion payoff and the deviation payoff such that there exists an interval to the right of $p^{*}$ such that collusion is easier to sustain for higher prices.

The derivative of the left hand side of (15) is zero, by construction of $p^{*}: \frac{\partial \pi\left(p^{*}\right)}{\partial p}=0$. The derivative of the right hand side equals:

$$
\widetilde{\beta}\left\{\left[\bar{q}\left(p^{*}\right)^{\prime} \tau(1-\beta)\right]-U_{\bar{\theta}}\left(p^{*}, f^{*}\right)^{\prime}\right\} .
$$


$U_{\bar{\theta}}$ is the information rent defined by:

$$
U_{\bar{\theta}}=u_{\bar{\theta}}\left(q_{\bar{\theta}}\left(p^{*}\right)\right)-p^{*} q_{\bar{\theta}}\left(p^{*}\right)-f^{*},
$$

where

$$
f^{*}=u_{\underline{\theta}}\left(q_{\underline{\theta}}\left(p^{*}\right)\right)-p^{*} q_{\underline{\theta}}\left(p^{*}\right),
$$

i.e. the low type's consumer surplus, hence,

$$
\frac{\partial f^{*}}{\partial p}=-q_{\underline{\theta}}\left(p^{*}\right)
$$

therefore,

$$
\frac{\partial U_{\bar{\theta}}}{\partial p}=-\left[q_{\bar{\theta}}\left(p^{*}\right)-q_{\underline{\theta}}\left(p^{*}\right)\right]<0 .
$$

Thus, (16) will be negative if:

$$
\begin{gathered}
\widetilde{\beta}\left\{[(1-\beta)] \tau\left[a q_{\bar{\theta}}^{\prime}+(1-\alpha) q_{\underline{\theta}}^{\prime}\right]+\left[q_{\bar{\theta}}\left(p^{*}\right)-q_{\underline{\theta}}\left(p^{*}\right)\right]\right\}<0 \\
\tau>\frac{q_{\bar{\theta}}\left(p^{*}\right)-q_{\underline{\theta}}\left(p^{*}\right)}{(1-\beta)\left[-a q_{\bar{\theta}}^{\prime}-(1-\alpha) q_{\underline{\theta}}^{\prime}\right]}=: \underline{\tau}
\end{gathered}
$$

which will be satisfied for $\tau$ sufficiently large.

However, for large values of $\tau$ it might become optimal for the deviator to serve only low types. Thus, we have to check that values of $\tau$ exist such that (17) holds and it is still optimal for the deviator to serve only high types. If $\beta \leq \min (\alpha, 1-\alpha)$, the deviator will either serve only low or only high types. If he serves high types only, his profit $\bar{\pi}$ is given by:

$$
\bar{\pi}=\beta\left[\tau(1-\beta)\left[\alpha q^{*}-\bar{q}\right]+u_{\bar{\theta}}(\bar{q})-U_{\bar{\theta}}\right],
$$

where

$$
U_{\bar{\theta}}=u_{\bar{\theta}}\left(\bar{q}^{*}\right)-p^{*} \bar{q}^{*}-\left(u_{\underline{\theta}}\left(\underline{q}^{*}\right)-p^{*} \underline{q}^{*}\right)
$$

where $p$ is defined by $(9), \bar{q}=q_{\bar{\theta}}(p), \underline{q}=q_{\underline{\theta}}(p)$, and $q^{*}=\alpha q_{\underline{\theta}}\left(p^{*}\right)+$ $(1-\alpha) q_{\underline{\theta}}\left(p^{*}\right)$, where $p^{*}$ is defined by $(1 \overline{3})$, and $U_{\bar{\theta}}$ is the high types information rent in the collusive outcome. Serving low types only yields:

$$
\underline{\pi}=\beta\left[\tau(1-\beta)\left[\alpha q^{*}-\underline{q}\right]+u_{\underline{\theta}}(\underline{q})\right] .
$$

Serving high types only therefore is optimal if $(18)>(19)$ :

$$
\tau<\frac{u_{\bar{\theta}}(\bar{q})-u_{\underline{\theta}}(\underline{q})-U_{\bar{\theta}}}{(\bar{q}-\underline{q})(1-\beta)}=: \bar{\tau} .
$$




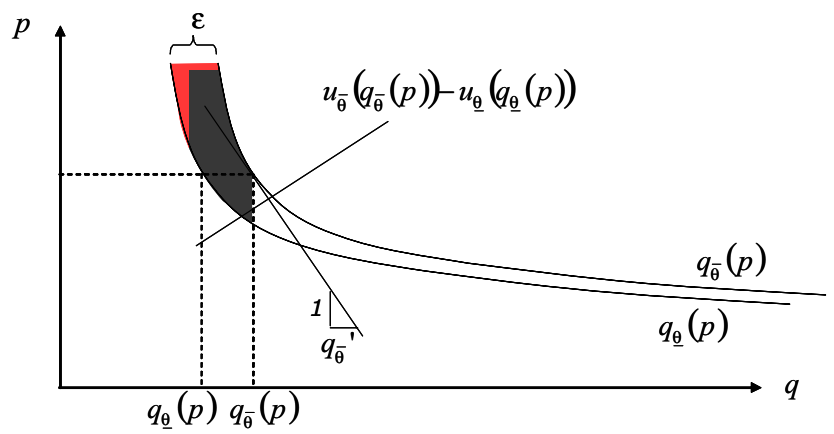

Figure 6: Illustration of (21)

The Interval $(\underline{\tau}, \bar{\tau})$ is non-empty if:

$$
\frac{q_{\bar{\theta}}\left(p^{*}\right)-q_{\underline{\theta}}\left(p^{*}\right)}{\left[-a q_{\bar{\theta}}^{\prime}-(1-\alpha) q_{\underline{\theta}}^{\prime}\right]}<\frac{u_{\bar{\theta}}(\bar{q})-u_{\underline{\theta}}(\underline{q})-U_{\bar{\theta}}}{(\bar{q}-\underline{q})},
$$

which is true for any values of $\alpha$ for (i) $\left|q_{\bar{\theta}}^{\prime}\right|$ and $\left|q_{\underline{\theta}}^{\prime}\right|$ sufficiently large, (ii) $u_{\bar{\theta}}(\bar{q})-u_{\underline{\theta}}(\underline{q})$ sufficiently large, and (iii) $q_{\bar{\theta}}(p)-q_{\underline{\theta}}(p)$ small. This can be satisfied for $q_{\bar{\theta}}(p)=q_{\underline{\theta}}(p)+\varepsilon$ and $q_{\underline{\theta}}(p)$ sufficiently convex. See Figure 6 for an illustration, where $u_{\bar{\theta}}(\bar{q})-u_{\underline{\theta}}(\underline{q})$ is the shaded area, which can be arbitrarily large.

\subsection{Proof of Lemma 3}

(i) Discrete case. Call $\left(q^{*}(\theta), t^{*}(\theta)\right)$ the profit maximizing contract. It is well known that in the optimum, the incentive compatibility constraint are "downward binding", i.e.:

$$
u_{\bar{\theta}}\left(q^{*}(\bar{\theta})\right)-t^{*}(\bar{\theta})=u_{\bar{\theta}}\left(q^{*}(\underline{\theta})\right)-t^{*}(\underline{\theta}) .
$$

Thus, if the contract would not include the offer $\left(q^{*}(\bar{\theta}), t^{*}(\bar{\theta})\right)$, type $\bar{\theta}$ would choose $\left(q^{*}(\underline{\theta}), t^{*}(\underline{\theta})\right)$. If this would increase profits, $\left(q^{*}(\theta), t^{*}(\theta)\right)$ would not be profit maximizing, a contradiction.

(ii) Continuum case. For the proof we can rely on well known characteristics of the solution to the adverse selection problem with a continuum of types, as presented e.g. in Fudenberg and Tirole (1991), Chapter 7.3. For 
the contract $\left(q^{*}(\theta), t^{*}(\theta)\right)$ to be implementable, we know that $q^{*}(\theta)$ must be non-decreasing, see Fudenberg and Tirole (1991), Theorem 7.2. The profit on a type $\theta$, denoted by $\pi_{\theta}$, is given by (see Fudenberg and Tirole (1991), p. 264; note that their valuation function $V_{0}(q, \theta)$ of the principal corresponds to our cost function $C(q)$ ):

$$
\pi_{\theta}=u\left(q^{*}(\theta)\right)-\int_{0}^{\theta} \frac{\partial u(q(\phi), \phi)}{\partial \phi} d \phi-C\left(q^{*}(\theta)\right) .
$$

The solution to the relaxed optimization problem of the monopolist (i.e. neglecting the non-monotonicity constraint $\frac{\partial q}{\partial \theta} \geq 0$ ) is given by (see Fudenberg and Tirole (1991), equation 7.12):

$$
\frac{\partial C}{\partial q}=\frac{\partial u}{\partial q}-\underbrace{\frac{1-P(\theta)}{p(\theta)} \cdot \frac{\partial^{2} u(q(\theta), \theta)}{\partial q \partial \theta}}_{:=z>0},
$$

where $P(\theta)$ denotes the cumulative density function of types and $p(\theta)$ denotes the density function. Using (24) to differentiate (23) with respect to the type yields:

$$
\begin{aligned}
\frac{\partial \pi_{\theta}}{\partial \theta} & =\frac{\partial u}{\partial q} \frac{\partial q}{\partial \theta}+\frac{\partial u}{\partial \theta}-\frac{\partial u}{\partial \theta}-\frac{\partial q}{\partial \theta}\left(\frac{\partial u}{\partial q}-z\right) \\
& =\frac{\partial q}{\partial \theta} z
\end{aligned}
$$

Thus, if the non-monotonicity constraint is not binding, $\frac{\partial \pi_{\theta}}{\partial \theta}>0$, i.e., profits are strictly increasing in types if $\frac{\partial q}{\partial \theta}>0$. If $\frac{\partial \pi_{\theta}}{\partial \theta}=0$ for some parameter region $[\underline{\theta}, \bar{\theta}]$, i.e. "bunching" occurs, profits will be the same for all types bunched under the same contract.

\subsection{Proof of Lemma 4}

(i) Due to the capacity constraint, the deviator serves high types only since profits are higher for higher types, as shown in Lemma 3. (ii) The deviator makes the same offer as the monopolist for the types he wants to serve: Generally, the optimum contract when serving all types is given by (Fudenberg and Tirole, 1991, 265):

$$
\frac{\partial v\left(q^{*}(\theta), \theta\right)}{\partial q}+\frac{\partial u\left(q^{*}(\theta), \theta\right)}{\partial q}-\frac{1-P(\theta)}{p(\theta)} \frac{\partial^{2} u\left(q^{*}(\theta), \theta\right)}{\partial q \partial \theta}=0,
$$


and

$$
t^{*}(\theta)=-u\left(q^{*}(\theta, \theta)\right)+\underbrace{\int_{0}^{\theta} \frac{\partial u\left(u^{*}\left(q^{*}(\tau), \tau\right)\right)}{\partial \tau} d \tau}_{I(\theta)},
$$

where $v()$ denotes the principal's valuation of the product (in our application, this is just equal to the production cost) and $I(\theta)$ is the information rent of type $\theta$. (25) defines the function $q^{*}(\theta)$, while (26) defines the profit maximizing payments for each type. Each type $\theta$, except the lowest, gets an information rent $I(\theta)$, which is increasing in $\theta$. In the solution to the deviator's problem, the marginal customer $\widetilde{\theta}$ must receive $I(\widetilde{\theta})$, as defined by $(26)$, in order to accept the deviator's offer. Substituting $I(\widetilde{\theta})$ into (26) shows that the optimum quantities $q^{*}(\theta)$ and optimum payments $t^{*}(\theta)$ for $\theta \geq \widetilde{\theta}$, do not depend on values of $\theta<\widetilde{\theta}$, and they therefore coincide with the monopolistic solution for these types.

\subsection{Proof of Proposition 4}

The deviator's payoff has two elements, the payments from customers, which we want to call $\Pi_{C}^{d e v}$, and the termination revenues, which we call $T$, which is the sum of termination revenues the deviator receives, minus the sum of termination fees he pays:

$$
\Pi_{\tau}^{d e v}=\Pi_{C}^{d e v}+T
$$

Denote by $\Pi^{d e v}$ the maximum deviation profit in the absence of termination fees $(\tau=0$; the outcome of Lemma 4$)$. We need to distinguish two cases to prove the proposition.

$T<0: T<0 \Longrightarrow \Pi_{\tau}^{d e v}<\Pi^{d e v}$, which follows trivially from the fact that the deviator can never receive more from the customers than $\Pi^{d e v}$. But if the deviator offers the same contract to the customers as the collusive contract, his termination revenues are strictly negative, since

$T(\widetilde{\theta}, \tau)=\tau(1-P(\widetilde{\theta}))[\underbrace{\int_{0}^{1} q(\theta) p(\theta) d \theta}_{\text {average incoming calls }}-\underbrace{\frac{1}{1-P(\widetilde{\theta})} \int_{\tilde{\theta}}^{1} q(\theta) p(\theta) d \theta}_{\text {average outgoing calls }}]<0$,

since $q(\theta)$ is increasing. 
$T \geq 0$ : Consider a contract $(\widetilde{q}(\theta), \widetilde{t}(\theta))$ that results in an equalized call balance for the deviator, i.e. the deviator has on average the same number of in- and outgoing calls per customer:

$$
(\widetilde{q}(\theta), \widetilde{t}(\theta)) \rightarrow T=0 \forall \tau>0 .
$$

Denote the resulting payments from customers, which, in this case, equal the deviator's total profit, by $\Pi(\widetilde{q}(\theta), \widetilde{t}(\theta))$. This is strictly lower than the profit of a deviator in the absence of termination fees, i.e. for $\tau=0$ :

$$
\Pi(\widetilde{q}(\theta), \widetilde{t}(\theta))<\Pi^{\operatorname{dev}} .
$$

In order to achieve an equalized call balance, the deviator either (i) serves only high types $\theta \geq \widetilde{\theta}$, but with a lower quantity, or (ii) serves also some low types $\theta<\widetilde{\theta}$. In either case, this reduces profits discretely and independent of the size of the termination fee $\tau$. For (i), customers will accept a lower $\widetilde{q}(\theta)$ only if also $\widetilde{t}(\theta)$ is strictly lower. Note that the decrease in quantity cannot be only marginal to achieve an equalized call balance, since the term in the square brackets in (28) is strictly negative. For (ii), the deviator must substitute some high types by low types in his customer pool. Even if he extracts the maximum profit from these types, this reduces profits compared to the case without termination fees, since, by Lemma 3, profits on lower types are lower.

By the same argument, any contract that will yield positive termination revenues for the deviator, $T>0$, will imply that the revenues from the customers will be even smaller than $\Pi(\widetilde{q}(\theta), \widetilde{t}(\theta))$ :

$$
\text { For } \tau>0: T>0 \rightarrow \Pi_{C}^{d e v}<\Pi(\widetilde{q}(\theta), \widetilde{t}(\theta)) .
$$

Again, the deviator must induce his customer pool to consume less. This he can achieve only by (i) lower $\widetilde{\widetilde{t}}(\theta)<\widetilde{t}(\theta)$ or (ii) by serving lower types.

Therefore, for all values of $\tau>0$,

$$
\Pi_{C}^{d e v}=\Pi^{d e v}-\Delta, \Delta>0 .
$$

Since $T$ approaches zero for $\tau \rightarrow 0$, there exists a cutoff value $\widehat{\tau}>0$, such that for $\tau<\widehat{\tau}$ we have that $T(\tau)<\Delta \rightarrow \Pi_{\tau}^{\text {dev }}=\Pi_{C}^{\text {dev }}+T<\Pi^{\text {dev }}$. Since the deviation profit is smaller for these low values of $\tau$ compared to $\tau=0$, and the punishment payoff for all future periods is left unaltered, deviation will now be no longer profitable for some low values of the discount factor $\delta$, and thereby facilitate collusion, as stated in the Proposition. 


\section{References}

Armstrong, M. (1998): "Network Interconnection in Telecommunications," Economic Journal, 108, 545-564.

(2002): "The Theory of Access Pricing and Interconncetion," in Handbook of Telecommunications Economics, ed. by M. E. Cave, S. K. Majumdar, and I. Vogelsang, pp. 295-384. Elsevier, Amsterdam.

Benoit, J.-P., and V. Krishna (1987): "Dynamic Duopoly: Prices and Quantities," Review of Economic Studies, 54, 23-35.

Competition Commission (2002): "Mobile Termination Charges," Reports on references under section 13 of the Telecommunications Act 1984 on the charges made by Vodafone, O2, Orange and T-Mobile for terminating calls from fixed and mobile networks.

Davidson, C., and R. Deneckere (1990): "Excess Capacity and Collusion," International Economic Review, 31(3), 521-541.

Dessein, W . (2004a): "Network Competition in Nonlinear Pricing," RAND Journal of Economics, 34(4), 593-611.

(2004b): "Network Competition with Hetorogenous Customers and Calling Patterns," Information Economics and Policy, 16, 323-245.

European Commission (2003): "Commission Recommendation of 11 February 2003 on Relevant Product and Service Markets Within the Electronic Communications Sector Susceptible to Ex Ante Regulation in Accordance with Directive (2002/21/EC) of the European Parliament and of the Council on a Common Regulatory Framework for Electronic Communication Networks and Services (2003/311/EC)," Official Journal of the European Union L114/45 (8.5.2003).

Fudenberg, D., and J. Tirole (1991): Game Theory. Mit Press, Cambridge, MA.

Gans, J. S., and S. P. King (2001): "Using 'Bill and Keep' Interconnect Arrangements to Soften Network Competition," Economics Letters, 71, 413-420.

Hahn, J.-H. (2004): "Network Competition and Interconnection with Heterogenous Subscribers," International Journal of Industrial Organization, $22,611-631$. 
IRG (2002): "Market Definition for Mobile Termination," Independent Regulators Group Working Paper.

(2004): "Market Data Analysis 2004 Report," .

Ivaldi, M., B. Jullien, P. Rey, P. Seabright, and J. Tirole (2003):

"The Economics of Tacit Collusion," Final report for DG Competition, European Commission.

Laffont, J.-J., P. Rey, and J. Tirole (1998a): "Network Competition: I. Overview and Nondiscriminatory Pricing," RAND Journal of Economics, 29(1), 1-37.

(1998b): "Network Competition: II. Price Discrimination," RAND Journal of Economics, 29(1), 38-56.

Laffont, J.-J., and J. Tirole (2000): Competition in Telecommunications. MIT Press, Cambridge, MA.

Mandy, D. M. (1992): "Nonuniform Bertrand Competition," Econometrica, 60(6), 1293-1330.

Ofcom (2003): "Ofcom Decision on Mobile Termination," http://www.ofcom.org.uk/consultations/past/wmvct/? $a=87101$.

Peitz, M., T. M. Valetti, and J. Wright (2004): "Competition in Telecommunications: An Introduction," Information Economics and Policy, 16, 315-321.

Vogelsang, I. (2003): "Price Regulation of Access to Telecommunications Networks," Journal of Economic Literature, 41(3), 830-862. 